\title{
XXIV.
}

\section{Die Abfassungszeit von Arrians Anabasis ${ }^{1}$.}

In der vor kurzem erschienenen Geschichte Alexanders des Großen und seiner Nachfolger von B. Niese, (Gotha 1893) ist auf Seite $8 \mathrm{Anm} .1$ ein Zweifel an der Richtigkeit der Ausführungen Nissens über die Abfassungszeit der Anabasis [Rhein. Mus. 43 (1888) 236 ff.] ausgesprochen, welcher sich durch eine Prüfung der Quellen begründen läßt. $\mathrm{Da}$ das Hauptergebniß Nissens sich nicht mit den von Alfred von Gutschmid in seinen Vorlesungen vorgetragenen Ansichten vereinigen ließ, versuchte ich schon vor längerer Zeit, diesen zu ihrem Rechte zu verhelfen. Das ist mir nicht völlig gelungen, aber ich machte einige Beobachtungen, die gegen Nissen sprachen. Eine derselben fand ich in einem 1890 erschienenen Werke eines italienischen Gelehrten (Bolla, Arriano di Nicomedia, Turin, Clausen) bestätigt. Da dieses Werk wenig Beachtung gefunden hat, sei es mir vergönnt, mit einigen Worten auf dasselbe hinzuweisen. Man wird dem Verfasser, welcher mit seinem Buche ein 'studio sintetico nel campo storico-letterario' über Arrian bieten wollte, Recht geben, wenn er von dem geringen Ertrage der Untersuchungen über den Ein-

1) Die folgenden Zeilen waren ein Excurs meiner im Jahre 1889 verfaßten und im Dec. 1890 bei der Tübinger philosopbischen Facultät eingereichten Dissertation über Plutarchs Vita Alexandrı Magni. Diese bildete das erste Capitel einer größeren Arbeit über die Quellen der Alexandersynoptiker. 
fluß des Merodot, Thukydides oder Xenophon auf die Kunstsprache des Arrian redet. Die sprachliche Untersuchung hätte meiner Meinung nach sich zunächst auf die Anabasis beschränken sollen und hätte dann in diesem Werke selbst interessante sprachliche Verschiedenheiten erwiesen, sie hätte ferner zeigen können, wie nahe Arrian sich mit Plutarch und Diodor beriihrt, d. h. wie unselbständig Arrian gewesen ist. Das Buch Bolla's ist zur einen Hälfte den äußeren Lebensverhältnissen Arrians, zur anderen seinen Werken gewidmet. Eine neue Sammlung der Fragmente Arrians --- das ist wohl die wichtigste Aufgabe der Arrianforschung - hat Bolla nicht veranstaltet. Die Frage, wo und in welcher Reihenfolge Arrian seine Schriften verfaßt habe, bietet manche Schwierigkeit. Bolla geht in seinem lesenswerthen Buche mit Sorgfalt auf diese Frage ein, allerdings ohne in der Weise die Beziehungen der Werke Arrians zu den Zeitverhältnissen zu würdigen, wie es Gutschmid in seinen Vorlesungen und Nissen in dem erwähnten Aufsatze, welchen Bolla nicht gekannt hat, gethan haben.

Nach Gutschmid gehört die Anabasis zu den früheren Schriften des Arrian und steht im Zusammenhange mit den Partherkriegen Trajans, durch welche das Interesse der Zeitgenossen wieder auf die Unternehmungen Alexanders gelenkt wurde. Mit diesem Werke

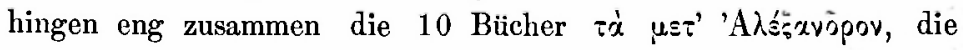
unverhältnißmäßig ausführlicher als die Anabasis waren ${ }^{2}$ ). Sie werden daher keine bloße Fortsetzung der Anabasis gewesen sein. Arrian wollte an einer classischen Behandlung der griechischen Geschichte den Nachweis fuihren, wie klug Hadrian daran gethan hatte, die unsinnigen Eroberungen Trajans aufzugeben. Man sieht, daß mit diesen Ansichten sich das Ergebniß des Aufsatzes Nissens, Arrian habe die Anabasis im hohen Alter verfaßt, nicht verträgt. Diese letztere Annahme läßt sich zwar nicht direkt widerlegen, die Behauptung Gutschmids nicht strikt beweisen, allein beweisen kann man, daß der von Nissen gefundene terminus post quem in Wirklichkeit ein terminus ante quem ist, und daß alle weiteren Folgerungen Nissens in dem genannten Aufsatze nichtig

2) Vgl. U. Köbler, Ueber die Diadochengeschichte A rrians. Sitzungs-Ber. d. Berliner Akad. (Phil.-hist. Cl.) 28 (1890) S. $557 \mathrm{ff}$. 
sind. Nissen versucht es, seine Behauptung durch einen doppelten Beweis zu stützen und hat auf Beziehungen zwischen Lukian und Arrian hingewiesen. Diese bestehen allerdings, nur muß der Spieß3 umgedreht werden: denn gleich die erste Voraus-

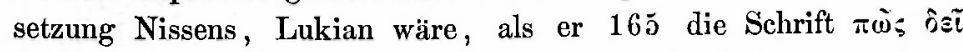

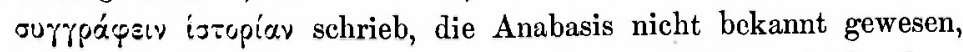
kann nicht bestehen. „Mag man noch so aufmerksam hinhorchen, wir werden zunächst nicht die leiseste Bezugnahme (auf die Anabasis) verspüren" sagt Nissen (a. a. O. S. 242). Es finden sich aber in dieser Schrift Lukians so deutliche Anspiclungen auf die Anabasis, dal3 jeder Zweifel ausgeschlossen ist. Wir dürfen die Anabasis nicht mit dem Maabe messen, welches der Verfasser an sein Werk legt. Arrian hat von sich und seinem Werke eine sehr hohe Meinung. Da er keinen Verleger hatte, der etwa auf die eigenartige und ungewöhnliche Auffassung des Autors aufmerksam machte, mußte Arrian es selbst thun und hat dabei ein wenig zu stark aufgetragen. Nicht bloß durch den Vergleich von Anab. VI 11, 8 mit Curtius Rufus IX 5, 21 ist Arrian gerichtet. Die hohen Anforderungen, die Lukian in seiner interessanten Schrift an den Geschichtschreiber stellt, hat Arrian in keiner Weise erfüllt. Nissen gesteht es zu, daB auch die Parthische Geschichte des Arrian gleich der Anabasis des schulmälbigen Einganges entbehrt haben könne, und daß Lukian hierauf anspiele. Das mag richtig sein, aber Lukian spielt direkt auf das Prooemium der Anabasis an, er hat wohl gewußt, was er von der naiven Begründung der Quellenauswahl in diesem Prooemium zu halten hatte. ",A Aristobul und Ptolemaios werden nicht gelogen haben, weil sie an den Feldzügen Alexanders Theil nahmen, und Ptolemaios war ja König, für den das Lügen eine Schmach gewesen wäre ${ }^{3}$ )“ “. Das schwächste Argument Arrians ist jedenfalls

8) Hübsch bemerkte Gutschmid dazu: „Als ob Könige nie gelogen hätten, wenn auch nicht alle so frecb wie Napoleon I". Micb haben die Worte Arrians a uf eine Vermuthung gebracht, die vielleicht die Abfassungszeit näber bestimmt. Enthalten sie nicht eine Ánspielung auf die Selbstbiographie Hadrians? Bei dem Cbarakter dieses Kaisers ist es scbwer anzunehmen, daß er stets der Wabrbeit die Ebre gegeben babe, und grade seine Persönlichkeit ist vielen Angriffen ausgesetzt und ihnen gegenüber meist wehrlos gewesen. Gegen einen litterarischen Angriff hat arrian mit jenen Worten die Selbstbiographie des Kaisers vertheidigen wollen. 




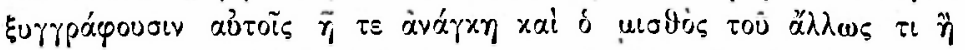

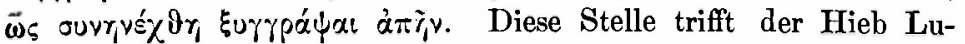

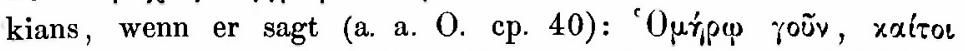

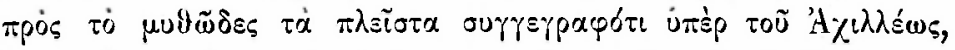

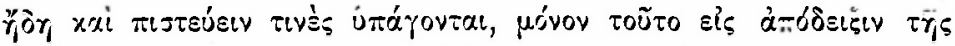

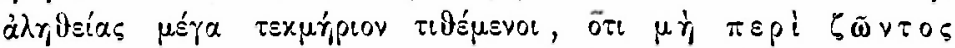

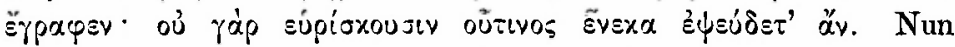
verstehen wir auch die Geschichte, welche Lukian cp. 12 von Aristobul erzählt, und die aller Ueberlieferung und dem Wesen des Aristobul widerspricht und nach dem Muster einer uns von dem Schwindler Onesikritos berichteten Episode (Plut. Alex. 46) erfunden ist. Während der Fahrt auf dem Hydaspes habe Aristobul nach Lukian Alexander ein Capitel aus seinem Opus vorgelesen und zwar eine Beschreibung eines Zweikampfes Alexanders mit Poros, in welchem Alexander einen Elephanten mit éinem Speerwurfe tödtet. Da habe Alexander dem Aristobul das Buch an den Kopf geworfen, es wäre ins Wasser gefallen, und

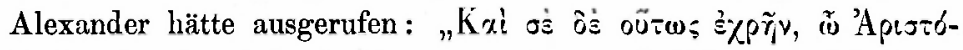

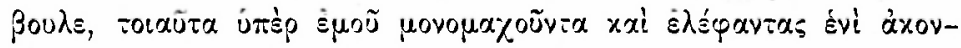
ii poveúovia. Mit Unrecht sagt Nissen: (a. a. O. S. 242) "Auf welchem Wege sie (d. h. diese Anckdote) dem leichtblütigen Journalisten zugeflogen sei, darf uns nicht beschäftigen: sicherlich jedoch hätte derselbe sich gehütet, mit falscher Gelehrsamkeit vor seinen Hörern und Lesern zu prunken, wenn er (was die Schrift Makrob. 22 bestätigt), aus den Eingangsworten Arrians gewußt hätte, daß der geschmähte Aristobul in hohem Greisenalter lange nach dem 'Tode des Königs seine Aufzeichnungen gemacht hat". Ich halte Lukian durchaus nicht für einen leichtblïtigen Journalisten, aber seit wann hüten diese Herren sich davor, selbst gegen besseres Wissen mit falscher Gelehrsamkeit zu prunken? Diese von Lukian aus den Fingern gesogene Geschichte hatte den Zweck, auch die 2te von Arrian im Prooemium erwähnte Hauptquelle lächerlich zu machen. Auch Nissen (a. a. O. S. 250) rechnet mit dem Factor, daß Lukian iubertrieb. So trägt er z. B. ein wenig stark auf in der 2ten auf die Anabasis bezüglichen Stelle, die auch Bolla (a. a. O. S. 9j Anm. 3) anführt, obne jedoch die Bedeutung dieser Parallele zu würdigen.

Ich setze zum besseren Verständniß das ganze Capitel (31) 
der Schrift Lukians her. Nachdem Lukian eine Reihe von Historiographen - zum Theil nennt er ihre Namen - gebrandmarkt hat, weil sie im Romanstile schrieben, fährt er fort: "Hor

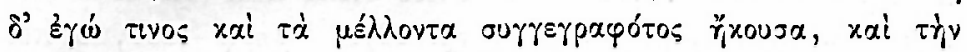

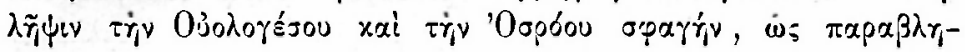

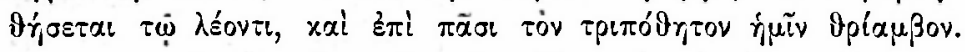

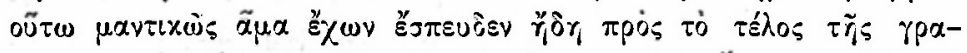

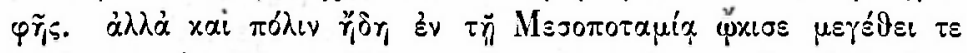



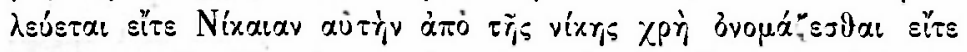

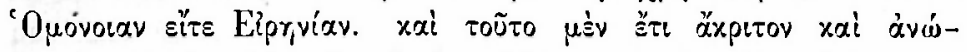

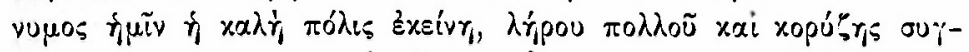

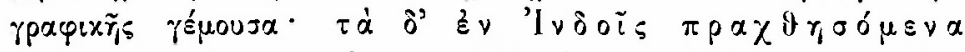

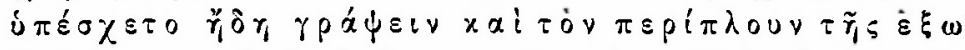

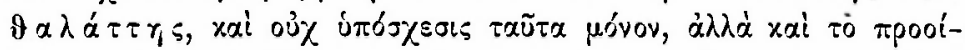

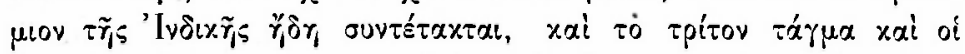

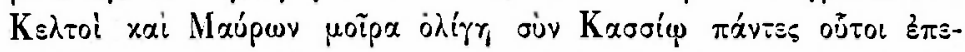

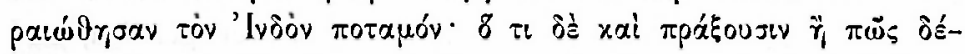

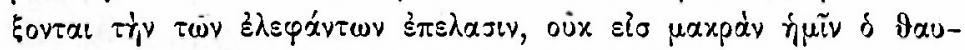

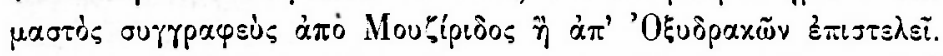

Am deutlichsten ist in diesem Passus die Anspielung auf die mehrfach in der Anabasis (V 6, 8; VI 16, 5; 28,6) ausgesprochene Absicht Arrians ein Werk über Indien zu schreiben. Aber auch das Uebrige läßt sich auf Arrian beziehen. Nissen (a. a. 0 . S. 250) nimmt an, daß im 27. Todtengespräch Lukian auf Arrians parthische Geschichte hinziele. Das mag der Fall sein, jedenfalls giebt Nissen zu, daß A rrian von Lukian verspottet wird, $\mathrm{da} B$ es ferner nicht zu bezweifeln ist, „daß Arrian wie die besten Kriegsschriftsteller früherer Zeiten Dinge erzählt haben wird, die einen starken Glauben verlangen und dem Vorwurf der Uebertreibung ausgesetzt sind". Mit gleichem Rechte können wir auf Arrians Parthergeschichte die Eingangsworte des angeführten Passus beziehen und ihnen vielleicht noch entnehmen, daß Arrian in seinem Werke sich nicht mit der Darstellung der zeitgenössischen Ereignisse begnügt habe, sondern den Waffen der Römer glänzende Erfolge im Oriente prophezeite. Als Lukian diese Schrift schrieb, (165) hatte der Siegeslauf der Römer im Oriente durch den Ausbruch einer Pest, dem der Friedensschluß folgte (Lukian cp. 15), schon sein Ende gefunden, und die spöttischen 
Worte Lukians treffen nicht nur den Jaupaotis surrpapsis Arrian, sondern auch die kriegerischen Unternehmungen der Römer, in welchen die Gedanken Trajans, der einst auf dem Euphrat beim Anblicke eines nach Indien segelnden Schiffes wehmüthig dem Makedonier Alexander den Inderzug neidete, wieder auflebten. Wenn mir der Nachweis gelungen ist, daß die erste Prämisse Nissens, in Lukians Schrift über die Geschichtschreibung finde sich keine Anspielung auf Arrians Anabasis, nicht bestehen kann, dann fallen auch die übrigen Argumente des doppelten Beweises. Sie stehen auch meist auf sehr schwachen Füßen. Ich greife nur 2 Beispiele heraus. Nissen (a. a. O. S. 253) ist der Meinung, daß Arrian An. V 7 bei der Besprechung des Brückenschlages nicht eigene Erfahrung wiedergebe, sondern einen 164 oder 165 durchgeführten Flußübergang im Auge habe. Woraus folgt das? Doch nicht aus der von Nissen angeführten

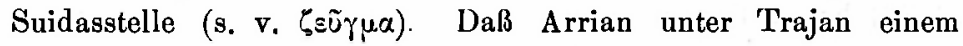
Brückenschlage größeren Stils hätte beiwohnen können, giebt Nissen zu. Daß er sich auf einen solchen bezieht ist auch die am nächsten liegende Annahme, der mit Recht Bolla (a. a. O. S. 17) gefolgt ist. Wenn Arrian An. VI 11, 2 einen Excurs über Arbela und Gaugamela bringt, so ziele er damit nach Nissen (a. a. O. S. 247) auf das 12. 'Todtengespräch Lukians, wo die Schlacht, wie stets in der Vulgata, nach dem Orte Arbela benannt wird. Das hieBe allerdings, um Nissens eigene Worte anzuführen, schweres Geschütz gegen Spatzen auffahren. Nissen hat übersehen, daß dieser Excurs sich auch bei Plut. Alex. 31 und Strabo pag. 737 findet, die ihn derselben Quelle wie Arrian (d. h. Agatharchides) entnahmen.

Einen terminus ante quem für die Abfassung der Anabasis hätten wir gefunden. Mir schien früher Anab. VII 27, 2 einen terminus post quem zu bieten, weil ich glaubte, daß der Autor, welchen Arrian dort geißelt, erst nach dem 'Tode des Antinoos geschrieben haben könne. Doch diese Vermuthung läßt sich nicht halten. Daß vorläufig der Einwand, nicht lange vor dem Jahre 165 könne Arrian die Anabasis verfaßt haben, weil sonst die Anspielungen Lukians ihre Spitze verlieren würden, berechtigt ist, weiß ich wohl. Zum Beweise dafür, daß die Anabasis in Athen verfaBt, führt Nissen (a. a. O. S. 239 An. 4) Anab. I 16,7 ; III 16,8 ; VI 11, 6 ; VII 13,5 ; 19, 2, Bolla (a. a. O. 
S. 75 A. 1) nur III 16,8 an. Nur diese Stelle könnte in Betracht kommen, aber auch sie beweist nur, daB Arrian, als er die Anabasis schrieb, Athen kannte.

Dal auch Lukian dem Arrian gerecht geworden ist, allerdings erst dem Todten, zeigt das 2te Capitel der Schrift Alexander oder der Trugprophet. Hier erwähnt Lukian eine Schrift Arrians über einen Räuberhauptmann Tilliboros, die eigentlich nicht in den Rahmen seiner Schriftstellerei hineinpaßt.

Gutschmid war der Meinung, dab diese kleine Monographie mit den bithynischen Studien Arrians zusammenhing. Sie sollte schwerlich blob dem Amüsement dienen, sondern ein so ernster Mann wie Arrian hätte sie geschrieben, um die Schäden der Verwaltung in der römischen Provinz aufzudecken. Das Strabos Geschichtswerk von Arrian in der Anabasis benutzt sei, hat vor Jahren Gutschmid vermuthet, allein in seine 'Geschichte Irans' (S. 73) hat er diese Vermuthung nicht aufgenommen. Sie wurde zum ersten Male von Kaerst in seiner Tübinger Dissertation (Beiträge zur Quellenkritik des Qu. Curtius Rufus, Gotha 1878) in Kurs gesetzt und ist allmählich Dogma geworden. Als solches erbt sie sich mit derselben Beharrlichkeit fort, mit der die Fabel vom Griechen Klitarch trotz des Gegenbeweises von Schoenle (Diodorstudien 1891) immer noch fortlebt. Seit der für die Quellenkritik der Alexandersynoptiker für immer grundlegenden Arbeit von A. Schoene (Analecta philologica historica I 1870) habe ich in der gesammten Litteratur nur éine richtige Andeutung der Quelle Arrians gefunden: In dem Vortrage über 'Isokrates und die griechische Geschichtschreibung' (Verhandlungen der 41. Philologenversammlung S. 118) redet Scala von einem Alexanderhistoriker, den er vorläufig namenlos lassen muß, aber einstweilen als Isokrateer bezeichnet, der in den hervorstechendsten Zügen bei Polybios, bei Justin zum Theil, bei Diodor in großem Auszuge, in Stücken bei A rrian vorliege. Wenn Scala auf dem mühevollen Wege, der zur Erkenntniß dieses Isokrateers führt, weiterschreiten wird, dann wird er gewahr werden, daß dem Bilde, welches er in seinen Studien des Polybios mit großer Liebe und vieler Mühe gezeichnet hat, die charakteristischen Grundlinien fehlen.

München.

Carl Erich Gleye. 